\title{
Imaging of enthesitis by an LED-based photoacoustic system (Erratum)
}

\author{
Janggun Jo, ${ }^{a, \dagger}$ Guan Xu, ${ }^{a, b, \dagger}$ Elena Schiopu, ${ }^{c}$ David Chamberland, ${ }^{c}$ \\ Girish Gandikota, ${ }^{\mathrm{d}}$ and Xueding Wang ${ }^{\mathrm{a}, \mathrm{d}}$ \\ ${ }^{a}$ University of Michigan, Department of Biomedical Engineering, Ann Arbor, Michigan, \\ United States \\ ${ }^{b}$ University of Michigan Medical School, Department of Ophthalmology and Visual Sciences, \\ Ann Arbor, Michigan, United States \\ ${ }^{c}$ University of Michigan Medical School, Division of Rheumatology, \\ Department of Internal Medicine, Ann Arbor, Michigan, United States \\ ${ }^{\mathrm{d}}$ University of Michigan Medical School, Department of Radiology, Ann Arbor, Michigan,
} United States

[DOI: $10.1117 / 1 . J B O .26 .5 .059801]$

This article [J. Biomed. Opt. 25(12), 126005 (2020) doi: 10.1117/1.JBO.25.12.126005 was originally published on 17 December 2020 with an error in the name of the first author. Janggun Jo was misspelled as "Junggun Jo." The error was corrected on 7 May 2021.

Address all correspondence to: Elena Schiopu, E-mail: eschiopu@med.umich.edu; Girish Gandikota, E-mail: ggirish@med.umich.edu; Xueding Wang, E-mail: xdwang@umich.edu

${ }^{\dagger}$ Equal contribution 